\title{
A characterization of the uniform rule without Pareto-optimality
}

\author{
Lars Ehlers
}

Received: 12 November 2010 / Accepted: 6 January 2011 / Published online: 22 February 2011

(C) The Author(s) 2011. This article is published with open access at SpringerLink.com

\begin{abstract}
We consider private good economies with single-peaked preferences. We show that the uniform rule is the only allocation rule satisfying $\Omega$-continuity, no-envy, and one-sided resource-monotonicity. This result strengthens a characterization of the uniform rule due to Thomson (Soc Choice Welf 11:205-223, 1994).
\end{abstract}

Keywords Uniform rule $\cdot$ Single-peaked preferences $\cdot \Omega$-continuity

JEL Classification D63 - D71

\section{Introduction}

We consider the problem of allocating a collective endowment among a set of agents. Each agent is equipped with a single-peaked preference relation meaning that there is a most preferred amount, called the peak, and preference is decreasing in either direction away from the peak. For instance, a group of workers have to provide a certain amount of working time in finishing a project. Each worker is proportionally paid according to the time he worked and they only receive their payments if the project was finished.

A rule is a systematic way of allocating the endowment among the agents. We are interested in identifying rules on the basis of desirable properties. If the amount of

I am grateful to an anonymous referee for his detailed comments and suggestions and to the SSHRC (Canada) and the FQRSC (Québec) for financial support.

L. Ehlers (®)

Département de Sciences Économiques and CIREQ,

Université de Montréal, Montréal, QC H3C 3J7, Canada

e-mail: lars.ehlers@umontreal.ca 
time for finishing the project changes, then the allotments of the workers have to be adjusted. We require that these adjustments are continuous with respect to the endowment $(\Omega$-continuity). If two economies only differ in their endowments and before and after the change the economy is in excess demand or in excess supply, then we require that all agents are affected in the same direction: either all workers weakly gain or they all weakly lose as a result of the change of the amount of working time (one-sided resource-monotonicity). Our last axiom postulates that each worker weakly prefers his working time to any working time allotted to another worker (no-envy). We show that the uniform rule is the only rule satisfying $\Omega$-continuity, no-envy, and one-sided resource-monotonicity. In contrast to most characterizations of the uniform rule our result does not use Pareto-optimality.

Sönmez (1994), Moulin (1999), and Ehlers (2002a,b) consider the implications of different resource-monotonicity requirements. Barberà et al. (1997) consider the implications of another solidarity property, namely when somebody's preference relation changes, then all other agents should be affected in the same direction. ${ }^{1}$

\section{The model and the result}

A collective endowment of a perfectly divisible good is allocated among a finite set of agents. Let $N=\{1, \ldots, n\}$ denote the finite set of agents. Each agent $i \in N$ is equipped with a preference relation $R_{i}$ over $\mathbb{R}_{+}$. Let $P_{i}$ denote the strict relation associated with $R_{i}$. The relation $R_{i}$ is single-peaked if there is a number $p\left(R_{i}\right) \in \mathbb{R}_{+}$, called the peak of $R_{i}$, such that for all $x, y \in \mathbb{R}_{+}$, if $x<y \leq p\left(R_{i}\right)$ or $p\left(R_{i}\right) \leq y<x$, then $y P_{i} x$. By $r_{i}(0)$ we denote the maximal amount which is weakly preferred under $R_{i}$ to receiving nothing, i.e. $r_{i}(0) \equiv \sup \left\{x \in \mathbb{R}_{+}: x R_{i} 0\right\}$. In general $r_{i}(0)$ might be infinite. A single-peaked preference relation $R_{i}$ is bounded if $r_{i}(0)<+\infty$. Let $\mathcal{R}$ denote the set of all bounded single-peaked preferences over $\mathbb{R}_{+}$. A (preference) profile $\left(R_{1}, \ldots, R_{n}\right) \in \mathcal{R}^{N}$ is denoted by $R$. An economy consists of a profile and an endowment, i.e. an economy is a tuple $(R, \Omega)$ where $R \in \mathcal{R}^{N}$ and $\Omega \in \mathbb{R}_{+}$. We do not allow free disposal and negative allotments. An admissible allocation for economy $(R, \Omega)$ is an $N$-vector $\left(x_{1}, \ldots, x_{n}\right) \in \mathbb{R}_{+}^{N}$ such that $\sum_{i \in N} x_{i}=\Omega$. An economy $(R, \Omega)$ is in excess demand if $\Omega \leq \sum_{i \in N} p\left(R_{i}\right)$ and $(R, \Omega)$ is in excess supply if $\sum_{i \in N} p\left(R_{i}\right) \leq \Omega$.

An (allocation) rule selects for each economy an admissible allocation. Formally, a rule is a mapping $\varphi: \mathcal{R}^{N} \times \mathbb{R}_{+} \rightarrow \mathbb{R}_{+}^{N}$ satisfying $\sum_{i \in N} \varphi_{i}(R, \Omega)=\Omega$ for all economies $(R, \Omega)$. We are interested in the following properties.

Pareto-optimality: For all $R \in \mathcal{R}^{N}$, all $\Omega \in \mathbb{R}_{+}$, and all admissible allocations $x$ for $(R, \Omega)$, if for some $i \in N, x_{i} P_{i} \varphi_{i}(R, \Omega)$, then for some $j \in N, \varphi_{j}(R, \Omega) P_{j} x_{j}$.

$\Omega$-continuity: For all $R \in \mathcal{R}^{N}, \varphi(R, \cdot)$ is continuous in $\Omega$.

\footnotetext{
1 See Ehlers (2002c) for another characterization of their class of rules.
} 
No-envy: For all $R \in \mathcal{R}^{N}$ and all $\Omega \in \mathbb{R}_{+}$, we have for all $i, j \in N, \varphi_{i}(R, \Omega) R_{i} \varphi_{j}$ $(R, \Omega)$.

One-sided resource-monotonicity: For all $R \in \mathcal{R}^{N}$, and all $\Omega, \Omega^{\prime} \in \mathbb{R}_{+}$such that $\Omega \leq \Omega^{\prime} \leq \sum_{i \in N} p\left(R_{i}\right)$ or $\Omega \geq \Omega^{\prime} \geq \sum_{i \in N} p\left(R_{i}\right)$, either [for all $i \in$ $N, \varphi_{i}(R, \Omega) R_{i} \varphi_{i}\left(R, \Omega^{\prime}\right)$ ] or [for all $i \in N, \varphi_{i}\left(R, \Omega^{\prime}\right) R_{i} \varphi_{i}(R, \Omega)$ ].

Benassy (1982) introduced the following rule.

Uniform Rule, $U$ : For all $(R, \Omega) \in \mathcal{R}^{N} \times \mathbb{R}_{+}$, (i) when $\Omega \leq \sum_{i \in N} p\left(R_{i}\right)$, for all $i \in N, U_{i}(R, \Omega)=\min \left\{p\left(R_{i}\right), \lambda\right\}$ where $\lambda$ solves $\sum_{i \in N} \min \left\{p\left(R_{i}\right), \lambda\right\}=\Omega$, and (ii) when $\sum_{i \in N} p\left(R_{i}\right) \leq \Omega$, for all $i \in N, U_{i}(R, \Omega)=\max \left\{p\left(R_{i}\right), \lambda\right\}$ where $\lambda$ solves $\sum_{i \in N} \max \left\{p\left(R_{i}\right), \lambda\right\}=\Omega$.

Sprumont (1991) characterized the uniform rule by Pareto-optimality, no-envy, and strategy-proofness (truth-telling is a weakly dominant strategy for each agent). ${ }^{2}$

Thomson (1994) showed that in Sprumont's characterization strategy-proofness can be replaced by the solidarity property one-sided resource-monotonicity. He shows in the proof of Theorem 2 (Part (i)) that Pareto-optimality and one-sided resourcemonotonicity imply $\Omega$-continuity.

Most characterizations of the uniform rule use Pareto-optimality. We offer a characterization which does not involve Pareto-optimality (and neither any anonymity requirement) and strengthens the characterization due to Thomson (1994).

Theorem 1 When all agents are only equipped with bounded single-peaked preferences, then the uniform rule is the only rule satisfying $\Omega$-continuity, no-envy, and one-sided resource-monotonicity.

The following examples show that the axioms in Theorem 1 are independent. In each of these examples Pareto-optimality is violated. Therefore, any two properties of Theorem 1 do not imply Pareto-optimality.

Example 1 Let $\bar{R} \in \mathcal{R}^{N}$ be such that for all $i \in N, \bar{R}_{i}=\bar{R}_{1}, p\left(\bar{R}_{1}\right)=1$, and $\bar{R}_{1}$ is symmetric around $p\left(\bar{R}_{1}\right)$ on [0,2]. For all $R \in \mathcal{R}^{N}$ and all $\Omega \in \mathbb{R}_{+}$, (i) if $(R, \Omega) \neq\left(\bar{R}, \frac{|N|}{2}+1\right)$, then $\varphi(R, \Omega)=U(R, \Omega)$, and (ii) if $(R, \Omega)=\left(\bar{R}, \frac{|N|}{2}+1\right)$, then $\varphi_{1}(R, \Omega) \equiv \frac{3}{2}$ and for all $i \in N \backslash\{1\}, \varphi_{i}(R, \Omega)=\frac{1}{2}$. The rule $\varphi$ satisfies no-envy and one-sided resource-monotonicity but not $\Omega$-continuity.

Example 2 For all $R \in \mathcal{R}^{N}$ and all $\Omega \in \mathbb{R}_{+}, \varphi_{1}(R, \Omega)=\Omega$ and for all $i \in N \backslash\{1\}$, $\varphi_{i}(R, \Omega)=0$. The rule $\varphi$ satisfies $\Omega$-continuity and one-sided resource-monotonicity but not no-envy.

Example 3 For all $R \in \mathcal{R}^{N}$, all $\Omega \in \mathbb{R}_{+}$, and all $i \in N, \varphi_{i}(R, \Omega)=\frac{\Omega}{|N|}$. The rule $\varphi$ satisfies $\Omega$-continuity and no-envy but not one-sided resource-monotonicity.

Note that Theorem 1 restricts the domain of single-peaked preferences by requiring them to be bounded. Let $\overline{\mathcal{R}}$ denote the full domain of single-peaked preferences.

$\overline{2 \text { Ching }(1992,1994) \text { and Ehlers }}$ (2000) further investigate this characterization. 
The following example shows (i) the characterization of Theorem 1 does not hold on the full domain of single-peaked preferences and (ii) there are other rules, different from the uniform rule, satisfying the properties of Theorem 1 on the full domain of single-peaked preferences.

Example 4 [Thomson (1994), Remark 2] Let $N=\{1,2\}$ and $\hat{R} \in \overline{\mathcal{R}}^{N}$ be such that $p\left(\hat{R}_{1}\right)=0, p\left(\hat{R}_{2}\right)=3$, and $\hat{r}_{2}(0)=+\infty$. For all $R \in \overline{\mathcal{R}}^{N}$ and all $\Omega \in \mathbb{R}_{+}$, (i) if $R=\hat{R}$ and $\Omega \geq 3$, then $\hat{\varphi}(R, \Omega)=\left(p\left(R_{1}\right), \Omega-p\left(R_{1}\right)\right)$, and (ii) otherwise $\hat{\varphi}(R, \Omega)=$ $U(R, \Omega)$. The rule $\hat{\varphi}$ satisfies $\Omega$-continuity, no-envy, and one-sided resource-monotonicity on the full domain but $\hat{\varphi} \neq U$.

Finally we note that different versions of one-sided resource-monotonicity have been proposed. A slightly stronger one is the following by Sönmez (1994).

Strong one-sided resource-monotonicity: For all $R \in \mathcal{R}^{N}$, and all $\Omega, \Omega^{\prime} \in \mathbb{R}_{+}$ such that $\Omega \leq \Omega^{\prime} \leq \sum_{i \in N} p\left(R_{i}\right)$ or $\Omega \geq \Omega^{\prime} \geq \sum_{i \in N} p\left(R_{i}\right)$, for all $i \in N$, $\varphi_{i}\left(R, \Omega^{\prime}\right) R_{i} \varphi_{i}(R, \Omega)$.

As Ehlers (2002a) points out, under Pareto-optimality, strong one-sided resourcemonotonicity and one-sided resource-monotonicity are equivalent.

Obviously, strong one-sided resource-monotonicity implies one-sided resourcemonotonicity and Theorem 1 remains valid when one-sided resource-monotonicity is replaced with strong one-sided resource-monotonicity. Not only this, (Ehlers 2002a, Theorem 4,) shows that after this replacement Theorem 1 is not tight because on the domain of bounded single-peaked preferences the uniform rule is the only rule satisfying no-envy and strong one-sided resource-monotonicity.

\section{Proof of Theorem 1}

Let $\varphi$ be a rule satisfying the properties of Theorem 1. First, we show that $\varphi$ selects the uniform allocation for all economies in excess demand.

Let $R \in \mathcal{R}^{N}$. Let $p^{1}(R)<p^{2}(R)<\cdots<p^{l}(R)$ be such that for all $k \in$ $\{1, \ldots, l\}, p^{k}(R) \in \mathbb{R}_{+}$, and $\left\{p^{k}(R): k \in\{1, \ldots, l\}\right\}=\left\{p\left(R_{i}\right): i \in N\right\}$. For all $k \in\{1, \ldots, l\}$, let $S_{k}=\left\{i \in N: p\left(R_{i}\right)=p^{k}(R)\right\}$. Obviously, $\left\{S_{k}: k \in\{1, \ldots, l\}\right\}$ is a partition of $N$. For all $t \in\{1, \ldots, l\}$, let $\beta^{k} \equiv \sum_{k=1}^{l}\left|S_{k}\right| \min \left\{p^{k}(R), p^{t}(R)\right\}$. We show by induction that for all $t \in\{1, \ldots, l\}$, for all $\Omega \in\left[0, \beta^{t}\right]$,

$$
\varphi(R, \Omega)=U(R, \Omega)
$$

Induction basis: For all $\Omega \in\left[0,|N| p^{1}(R)\right]$ and all $i \in N, \varphi_{i}(R, \Omega)=\frac{\Omega}{|N|}$.

Proof of induction basis: Suppose that for some $\Omega^{\prime} \in\left[0,|N| p^{1}(R)\right]$ and some $j \in N$, $\varphi_{j}\left(R, \Omega^{\prime}\right)>p^{1}(R)$. Since by definition for all $i \in N, \varphi_{i}(R, 0)=0$, then $\Omega$-continuity implies that for some $\Omega^{\prime \prime} \in\left(0, \Omega^{\prime}\right), \varphi_{j}\left(R, \Omega^{\prime \prime}\right)=p^{1}(R)$. Thus, by no-envy, for all $i \in N, \varphi_{i}\left(R, \Omega^{\prime \prime}\right) \geq p^{1}(R)$. Hence, $\sum_{i \in N} \varphi_{i}\left(R, \Omega^{\prime \prime}\right) \geq|N| p^{1}(R)>\Omega^{\prime \prime}$, a contradiction. 
Therefore, for all $\Omega \in\left[0,|N| p^{1}(R)\right]$ and all $i \in N, \varphi_{i}(R, \Omega) \leq p^{1}(R)$. By no-envy, for all $i \in N, \varphi_{i}(R, \Omega)=\frac{\Omega}{|N|}$, the desired conclusion.

Suppose that (1) holds for $t \in\{1, \ldots, l-1\}$.

Induction step: (1) holds for $t+1$.

Proof of induction step: First, we show the following claim.

Claim A: For all $\Omega \in\left(\beta^{t}, \beta^{t+1}\right]$ and all $i \in \cup_{k=1}^{t} S_{k}, \varphi_{i}(R, \Omega) \in\left[0, p\left(R_{i}\right)\right]$.

Proof of Claim A: Suppose that for some $\Omega^{\prime} \in\left(\beta^{t}, \beta^{t+1}\right]$, some $k \in\{1, \ldots, t\}$, and some $j \in S_{k}, \varphi_{j}\left(R, \Omega^{\prime}\right)>p\left(R_{j}\right)$. If $\varphi_{j}\left(R, \Omega^{\prime}\right)>p^{l}(R)$, then $\Omega$-continuity implies that for some $\Omega^{\prime \prime} \in\left[0, \Omega^{\prime}\right), \varphi_{j}\left(R, \Omega^{\prime \prime}\right)=p^{l}(R)$. Since (1) holds for $t$, it follows that $\Omega^{\prime \prime} \in\left(\beta^{t}, \beta^{t+1}\right)$. Thus, without loss of generality, we may suppose that $p\left(R_{j}\right)<\varphi_{j}\left(R, \Omega^{\prime}\right) \leq p^{l}(R)$.

By no-envy, the previous fact, and single-peakedness, for all $i \in S_{l}, \varphi_{i}\left(R, \Omega^{\prime}\right) R_{i} \varphi_{j}$ $\left(R, \Omega^{\prime}\right) P_{i} p\left(R_{j}\right)$. Since $k \leq t$, we have by the induction hypothesis, $\varphi\left(R, \beta^{k}\right)=$ $U\left(R, \beta^{k}\right)$. Thus, $\varphi_{j}\left(R, \beta^{k}\right)=U_{j}\left(R, \beta^{k}\right)=p\left(R_{j}\right)<\varphi_{j}\left(R, \Omega^{\prime}\right)$. Hence, $\varphi_{j}\left(R, \beta^{k}\right)$ $P_{j} \varphi_{j}\left(R, \Omega^{\prime}\right)$ and for all $i \in S_{l}, \varphi_{i}\left(R, \Omega^{\prime}\right) P_{i} \varphi_{i}\left(R, \beta^{k}\right)$. Because $\beta^{k}, \Omega^{\prime} \leq \sum_{i \in N} p\left(R_{i}\right)$, this contradicts one-sided resource-monotonicity.

Let $\bar{\Omega} \in\left(\beta^{t}, \beta^{t+1}\right]$. Next we show that for all $i \in \cup_{k=1}^{t} S_{k}$,

$$
\varphi_{i}(R, \bar{\Omega})=p\left(R_{i}\right)
$$

Suppose that for some $\bar{k} \in\{1, \ldots, t\}$ and some $j \in S_{\bar{k}}, \varphi_{j}(R, \bar{\Omega}) \neq p\left(R_{j}\right)$. Thus, by Claim A, $\varphi_{j}(R, \bar{\Omega})<p\left(R_{j}\right)$, and, by no-envy, for all $i \in N, \varphi_{i}(R, \bar{\Omega}) \neq p\left(R_{j}\right)$. If for all $i \in N, \varphi_{i}(R, \bar{\Omega})<p\left(R_{j}\right)$, then using Claim A, for all $i \in N, \varphi_{i}(R, \bar{\Omega}) \leq$ $\min \left\{p\left(R_{i}\right), p\left(R_{j}\right)\right\}$. Hence, $\sum_{i \in N} \varphi_{i}(R, \bar{\Omega}) \leq \sum_{i \in N} \min \left\{p\left(R_{i}\right), p\left(R_{j}\right)\right\} \leq \beta^{t}<\overline{\bar{\Omega}}$, a contradiction.

Thus, for some $h \in N, \varphi_{h}(R, \bar{\Omega})>p\left(R_{j}\right)$. If $\varphi_{h}(R, \bar{\Omega}) \leq p^{l}(R)$, then by noenvy, for all $i \in S_{l}, \varphi_{i}(R, \bar{\Omega}) R_{i} \varphi_{h}(R, \bar{\Omega}) P_{i} p\left(R_{j}\right)$. Hence, by $\bar{k} \leq t$ and the induction hypothesis, $\varphi\left(R, \beta^{\bar{k}}\right)=U\left(R, \beta^{\bar{k}}\right)$. Hence, by $\varphi_{j}\left(R, \beta^{\bar{k}}\right)=p\left(R_{j}\right) \neq \varphi_{j}(R, \bar{\Omega})$, we have $\varphi_{j}\left(R, \beta^{\bar{k}}\right) P_{j} \varphi_{j}(R, \bar{\Omega})$ and for all $i \in S_{l}, \varphi_{i}(R, \bar{\Omega}) P_{i} \varphi_{i}\left(R, \beta^{\bar{k}}\right)$. Because $\beta^{\bar{k}}, \bar{\Omega} \leq \sum_{i \in N} p\left(R_{i}\right)$, this contradicts one-sided resource-monotonicity.

Hence, we are left with the case that for some $h \in N, \varphi_{h}(R, \bar{\Omega})>p^{l}(R)$. By $\Omega$-continuity, for some $\tilde{\Omega} \in[0, \bar{\Omega}), \varphi_{h}(R, \tilde{\Omega})=p^{l}(R)$. By no-envy, for all $i \in$ $S_{l}, \varphi_{i}(R, \tilde{\Omega})=p^{l}(R)$. If $\tilde{\Omega} \in\left[0, \beta^{t}\right]$, then the previous facts contradict the induction hypothesis. Thus, $\tilde{\Omega}>\beta^{t}$. By no-envy, for all $i \in N, \varphi_{i}(R, \tilde{\Omega}) \leq p^{l}(R)$, and, by Claim A, for all $i \in \cup_{k=1}^{t} S_{k}, \varphi_{i}(R, \tilde{\Omega}) \leq p\left(R_{i}\right)$. Suppose that for some $k \in\{1, \ldots, t\}$ and some $j \in S_{k}, \varphi_{j}(R, \tilde{\Omega})<p\left(R_{j}\right)$. By the induction hypothesis, $\varphi\left(R, \beta^{k}\right)=$ $U\left(R, \beta^{k}\right)$. Hence, $\varphi_{j}\left(R, \beta^{k}\right) P_{j} \varphi_{j}(R, \tilde{\Omega})$ and for all $i \in S_{l}, \varphi_{i}(R, \tilde{\Omega}) P_{i} \varphi_{i}\left(R, \beta^{k}\right)$. Because $\beta^{k}, \tilde{\Omega} \leq \sum_{i \in N} p\left(R_{i}\right)$, this contradicts one-sided resource-monotonicity. Hence, for all $i \in \cup_{k=1}^{t} S_{k}, \varphi_{i}(R, \tilde{\Omega})=p\left(R_{i}\right)$. 
If $t+1=l$, then for all $i \in N, \varphi_{i}(R, \tilde{\Omega})=p\left(R_{i}\right)$. Thus, $\sum_{i \in N} \varphi_{i}(R, \tilde{\Omega})=$ $\sum_{\in N} p\left(R_{i}\right)=\beta^{l} \geq \bar{\Omega}>\tilde{\Omega}$, a contradiction.

If $t+1<l$, then by $\tilde{\Omega}<\beta^{t+1}$, for some $k \in\{t+1, t+2, \ldots, l-1\}$ and some $j \in S_{k}, \varphi_{j}(R, \tilde{\Omega})<p^{k}(R)$. Let $i \in S_{l}$. By $\Omega$-continuity and $\varphi_{i}(R, \tilde{\Omega})=p^{l}(R)$, for some $\hat{\Omega} \in[0, \tilde{\Omega}), \varphi_{i}(R, \hat{\Omega})=p^{k}(R)$. By no-envy and $p\left(R_{j}\right)=p^{k}(R), \varphi_{j}(R, \hat{\Omega})=$ $p^{k}(R)$.

Thus, $\varphi_{j}(R, \hat{\Omega}) P_{j} \varphi_{j}(R, \tilde{\Omega})$ and $\varphi_{i}(R, \tilde{\Omega}) P_{i} \varphi_{i}(R, \hat{\Omega})$. Because $\hat{\Omega}, \tilde{\Omega} \leq \sum_{i \in N}$ $p\left(R_{i}\right)$, this contradicts one-sided resource-monotonicity.

Therefore, (2) is true. Now similar arguments as in the proof of the induction basis show that for all $i \in \cup_{k=t+1}^{l} S_{k}, \varphi_{i}(R, \Omega)=U_{i}(R, \Omega)$, the desired conclusion.

Let $\Omega \in \mathbb{R}_{+}$be such that $(R, \Omega)$ is in excess demand. Then $\Omega \leq \sum_{i \in N} p\left(R_{i}\right)=\beta^{l}$ and by (1), $\varphi(R, \Omega)=U(R, \Omega)$, the desired conclusion for all economies in excess demand. Let $\bar{r}=\max \left\{r_{i}(0): i \in N\right\}$. Since for all $i \in N, R_{i}$ is bounded, we have $\bar{r}<+\infty$. For economies in excess supply, we make the following observations.

First, it follows from no-envy and the definition of $\bar{r}$ that for all $\Omega \in[0,|N| \bar{r}]$ and all $i \in N, \varphi_{i}(R, \Omega) \in[0, \bar{r}]$.

Second, for all $\Omega \in(|N| \bar{r},+\infty)$ and all $i \in N, \varphi_{i}(R, \Omega)=\frac{\Omega}{|N|}$. Suppose the previous statement is not true for $\Omega^{\prime} \in(|N| \bar{r},+\infty)$. Then for some $i, j \in N, \varphi_{j}\left(R, \Omega^{\prime}\right)<$ $\varphi_{i}\left(R, \Omega^{\prime}\right)$ and $\bar{r}<\varphi_{i}\left(R, \Omega^{\prime}\right)$, contradicting no-envy.

Using the first observation, for all $\Omega \in\left[\sum_{i \in N} p\left(R_{i}\right),|N| \bar{r}\right]$ we apply the same arguments as for economies in excess demand to deduce that $\varphi(R, \Omega)=U(R, \Omega)$. Then by the second observation it follows that $\varphi$ selects the uniform allocation for all economies in excess supply, the desired conclusion.

Open Access This article is distributed under the terms of the Creative Commons Attribution License which permits any use, distribution and reproduction in any medium, provided the original author(s) and source are credited.

\section{References}

Barberà S, Jackson M, Neme A (1997) Strategy-proof allotment rules. Games Econ Behav 18:1-21

Benassy JP (1982) The economies of market disequilibrium. Academic Press, San Diego

Ching S (1992) A simple characterization of the uniform rule. Econ Lett 40:57-60

Ching S (1994) An alternative characterization of the uniform rule. Soc Choice Welf 11:131-136

Ehlers L (2000) Indifference and the uniform rule. Econ Lett 67:303-308

Ehlers L (2002a) Resource-monotonic allocation when preferences are single-peaked. Econ Theory 20:113-131

Ehlers L (2002b) On fixed-path rationing methods. J Econ Theory 106:472-477

Ehlers L (2002c) Strategy-proof allocation when preferences are single-plateaued. Rev Econ Design 7:105115

Moulin H (1999) Rationing a commodity along fixed paths. J Econ Theory 84:41-72

Sönmez T (1994) Consistency, monotonicity, and the uniform rule. Econ Lett 46:229-235

Sprumont Y (1991) The division problem with single-peaked preferences: a characterization of the uniform allocation rule. Econometrica 59:509-519

Thomson W (1994) Resource-monotonic solutions to the problem of fair division when preferences are single-peaked. Soc Choice Welf 11:205-223 